\title{
Adición del Antioxidante Tempol en la Criopreservación de Espermatozoides de Alpaca (Vicugna pacos) Colectados por Desviación del Conducto Deferente
}

\author{
Addition of Antioxidant Tempol in Cryopreservation of Alpaca (Vicugna pacos) \\ SPerm Collected by Deviation of the Vas Deferens
}

Oscar E. Gómez-Quispe ${ }^{1,4}$, Manuel Guido Pérez³, Virgilio Machaca M.²

\section{Resumen}

El presente estudio tuvo como objetivo determinar el efecto de la adición del antioxidante Tempol en la motilidad individual y progresiva a la refrigeración y descongelación de espermatozoides de alpaca. Para ello, se desvió el conducto deferente de tres alpacas con adecuados parámetros seminales y los espermatozoides fueron colectados tres veces por semana y mezclados para anular el efecto individual. La muestra colectiva se dividió en cuatro alícuotas y fueron colocadas en refrigeración hasta alcanzar $5^{\circ} \mathrm{C}$ en $2 \mathrm{~h}$. El antioxidante fue añadido en concentraciones de $0,0.5,1.0$ y $1.5 \mathrm{mM}$ cuando las alícuotas alcanzaron los $10^{\circ} \mathrm{C}$. Las muestras fueron envasadas en pajillas de $0.25 \mathrm{ml}$, colocadas sobre vapores de nitrógeno líquido por 6 min y sumergidas. La motilidad individual de refrigeración fue 55.1, 63.7, 52.8 y 45.0 ( $\mathrm{p}<0.05)$ y a la descongelación 35.7, $42.3,29.0$ y $18.1 \%$ ( p<0.05) para $0,0.5,1.0$ y $1.5 \mathrm{mM}$ de Tempol, respectivamente. La motilidad progresiva a la refrigeración fue 36.0, 42.4, 28.4 y $21.7(\mathrm{p}<0.05)$ y a la descongelación $24.4,27.3,21.1$ y $15.8 \%$ para $0,0.5,1.0$ y $1.5 \mathrm{mM}$ de Tempol, respectivamente $(\mathrm{p}<0.05)$. La adición de una baja concentración de antioxidante Tempol mejoró la motilidad individual y progresiva en la criopreservación de espermatozoides provenientes del conducto deferente de la alpaca.

Palabras clave: motilidad, semen, camélidos, refrigeración, congelación, descongelación

\footnotetext{
${ }^{1}$ Laboratorio de Reproducción Animal, ${ }^{2}$ Quirófano Veterinario, Facultad de Medicina Veterinaria y Zootecnia, Universidad Nacional Micaela Bastidas de Apurímac, Perú

${ }^{3}$ Laboratorio de Reproducción Animal, Facultad de Medicina Veterinaria y Zootecnia, Universidad Nacional del Altiplano, Puno, Perú

${ }^{4}$ E-mail: oegomezq@hotmail.com
}

Recibido: 14 de abril de 2015

Aceptado para publicación: 2 de diciembre de 2015 
The aim of this study was to determine the effect of adding antioxidant Tempol in the individual and progressive motility of alpaca sperm at cooling and after thawing. Previously, the vas deferens was deviated in three males with suitable semen parameters. Sperm were collected three times per week and pooled to avoid the individual effect. The sample was distributed in four aliquots and placed in refrigeration to reach $5^{\circ} \mathrm{C}$ in $2 \mathrm{~h}$. The antioxidant was added in four concentrations: $0,0.5,1.0$ y $1.5 \mathrm{mM}$ when the aliquots reached $10{ }^{\circ} \mathrm{C}$. The sperm were filled in $0.25 \mathrm{ml}$ straws, placed over liquid nitrogen vapor by $6 \mathrm{~min}$ and then submerged. Individual motility at cooling was 55.1, 63.7, 52.8 and 45.0 $(\mathrm{p}<0.05)$ and after thawing 35.7, 42.3, 29.0 and $18.1 \%(\mathrm{p}<0.05)$ for $0,0.5,1.0$ and $1.5 \mathrm{mM}$ Tempol, respectively. Progressive motility at cooling was 36.0, 42.4, 28.4 and $21.7(\mathrm{p}<0.05)$ and after thawing $24.4,27.3,21.1$ and $15.8 \%$ for $0,0.5,1.0$ y $1.5 \mathrm{mM}$ Tempol, respectively $(\mathrm{p}<0.05)$. The individual and progressive motility was improved by addition of a low concentration of Tempol antioxidant $(0.5 \mathrm{mM})$ during the criopreservation of alpaca sperms harvested by vas deferens.

Key words: motility, semen, camelids, refrigeration, freezing, thawing

\section{INTRODUCCIÓN}

La inseminación artificial como técnica reproductiva en los camélidos sudamericanos no ha sido desarrollada completamente, debido a la baja eficiencia de los procedimientos rutinarios existentes para el congelamiento de semen de estas especies. En la alpaca (Vicugna pacos), el semen descongelado tiene una baja motilidad (20\%) (Santiani et al., 2005,2013 ), atribuido entre otros factores, a la alta viscosidad del semen, que ha dificultado grandemente su manipulación y procesamiento (von Baer y Hellemann, 1998). Esto ha propiciado diversas investigaciones, incluyendo el desarrollo de la técnica alternativa de colección de espermatozoides desprovistos de plasma seminal del conducto deferente (Quintano, 2002, Pérez et al., 2006, 2014). El empleo de esta técnica ha permitido conseguir una mejora en la motilidad individual (41\%) a la descongelación (Deza, 2004).

Uno de los factores de la baja motilidad de los espermatozoides de la alpaca se atribuye al estrés oxidativo del semen, debido a la producción de especies reactivas de oxigeno (ROS) durante la criopreservación, originando la peroxidación de las membranas espermáticas que afectan la organización y función de la membrana del espermatozoide (Tortolero et al., 2005), lo que conllevaría a la disminución de la motilidad y viabilidad espermática (Santiani, 2001, 2014). Al respecto, se ha indicado que la membrana del espermatozoide sufre daños en el proceso de congelación (Parks y Graham, 1992) por los cambios de temperatura que produce el shock térmico, la toxicidad de los crioprotectores y la formación-disolución de hielo en el medio extracelular (Rota et al., 1997).

Entre otros, el antioxidante Tempol (4hydroxy-2,2,6,6-tetramethylpiperidine-1-oxyl), ha sido empleado para disminuir el efecto dañino del ROS, el cual previene la pérdida de motilidad y viabilidad espermática durante la refrigeración de los espermatozoides de ovino (Mara et al., 2005; Ruiz et al., 2007; Sandoval et al., 2007) y equino (Ball et al., 2001), aunque con resultados contradictorios. También se ha empleado en el congelamiento de espermatozoides de bovino (Foote et al., 2002) y alpacas (Santiani et al., 2005, 2012, 2013). 
Por otro lado, existe limitada información sobre la motilidad progresiva en semen de camélidos. Esto es debido, en parte, a la característica viscosa del semen colectado por vagina artificial (Sumar, 1996), que ocasiona que la motilidad espermática sea oscilatoria y no progresiva (Bravo et al., 2000). Según Garnica et al. (1993), la viscosidad afectaría la motilidad del espermatozoide. En estudios previos, la obtención de espermatozoides desprovistos de plasma seminal por la técnica de la desviación del conducto deferente permite observar una motilidad progresiva.

El presente estudio pretende contribuir en las bases de un protocolo de criopreservación de rutina del semen de la alpaca, que permita el uso eficiente de los reproductores y, consecuentemente, intensificar el mejoramiento genético. Es así que se tuvo como objetivo determinar el efecto de la adición del antioxidante Tempol en la motilidad individual y la motilidad progresiva a la refrigeración y congelación de espermatozoides de alpaca colectados del conducto deferente.

\section{Materiales y Métodos}

\section{Localización del Estudio}

El estudio se llevó a cabo en el Laboratorio de Reproducción Animal de la Universidad Nacional Micaela Bastidas de Apurímac (UNAMBA), ubicado en el distrito y provincia de Abancay, departamento de Apurímac, Perú, a $2378 \mathrm{~m}$ de altitud, donde la temporada de lluvias es de noviembre a marzo y la temperatura máxima media y mínima media es de 23.8 y $11.7^{\circ} \mathrm{C}$, respectivamente.

\section{Animales}

Para la investigación se tomó una muestra por conveniencia, consistente de tres alpacas machos que tenían como motilidad progresiva mayor a 50\%, según lo propuesto por Niasari-Naslaji et al. (2007). Los animales fueron trasladados del Centro Poblado de San Miguel de Mestizas, distrito de Iscahuaca (Cotaruse, Apurímac) al Campus de la UNAMBA, donde fueron sometidos a un periodo de adaptación durante ocho meses, alimentados con pastos naturales y suplementados con $2 \mathrm{~kg}$ de alfalfa por día.

\section{La Técnica Quirúrgica}

El proceso preoperatorio, la técnica quirúrgica de desviación del conducto deferente y el tratamiento posoperatorio, que conllevaron a las fístulas en la cara interna de los muslos de la alpaca, se realizaron acorde con Pérez et al. (2006).

\section{Colección y Procesamiento de Semen}

La colección de espermatozoides se realizó tres veces por semana, luego de acostumbrar a los animales. Para la colección, los animales fueron derribados, la cara interna del muslo fue higienizada, y a través de suaves masajes se facilitó la salida de los espermatozoides por la abertura del conducto deferente. Los espermatozoides fueron aspirados con una jeringa de tuberculina y vertidos a un tubo colector que contenía 0.5 $\mathrm{ml}$ de dilutor Tris (Pérez et al., 2006). Las muestras de los tres machos fueron mezcladas en un solo tubo para anular el efecto individual, que se colocó dentro de un vaso de precipitación atemperado en baño María a $37^{\circ} \mathrm{C}$. Se evaluó la motilidad individual y progresiva del momento inicial. En ambos casos se tomaron 200 espermatozoides para realizar el contaje, para lo cual se utilizó un microscopio con salida de imagen a pantalla de computadora con una magnificación de 400X (BA210, Clinical MOTIC, China Group Ltd). Se determinó también la concentración espermática a través de un hemocitometro (cámara de Neubauer). 


\section{Refrigeración}

Las muestras colectivas de espermatozoides, mantenidas a $37{ }^{\circ} \mathrm{C}$, fueron diluidas con el dilutor (Tris $2.42 \mathrm{~g}$, ácido cítrico dihidratado $1.34 \mathrm{~g}$, fructosa $1 \mathrm{~g}$, agua bidestilada $100 \mathrm{ml}$, estreptomicina $33 \mathrm{mg}$, lincomicina $16.5 \mathrm{mg}$, gentamicina $27.5 \mathrm{mg}$, tilosina $5.5 \mathrm{mg}$ ) y $20 \% \mathrm{v} / \mathrm{v}$ de yema de huevo, en una proporción de 1:2 (muestra:dilutor). En seguida se dividieron en cuatro alícuotas que fueron llevadas a refrigeración para que alcancen una temperatura de $5^{\circ} \mathrm{C}$ en un tiempo de 2 h (Deza 2004; Pérez et al., 2006). El antioxidante Tempol (4-hydroxy-2,2,6,6tetramethylpiperidine-1-oxyl, Sigma-Aldrich, EEUU), se añadió en concentraciones de 0 , $0.5,1.0$ y $1.5 \mathrm{mM}$ cuando las alícuotas alcanzaron los $10^{\circ} \mathrm{C}$ (Santiani et al., 2013) y se siguió enfriando hasta $5{ }^{\circ} \mathrm{C}$. La motilidad individual y progresiva fue evaluada cuando el semen diluido alcanzó los $5{ }^{\circ} \mathrm{C}$.

\section{Congelación/Descongelación}

Seguidamente se agregó $7.5 \%$ v/v de glicerol a las alícuotas (Deza, 2004). Se llevó las muestras a un periodo de estabilización de $30 \mathrm{~min}$. Pajillas de $0.25 \mathrm{ml}$ se llenaron con la dilución de espermatozoides, siendo ajustadas previamente a una dosis de 20 millones de espermatozoides con motilidad progresiva. Las pajillas se sellaron con alcohol polivinílico y se colocaron sobre vapores de nitrógeno líquido en una rejilla de congelación dentro de una caja de tecnopor (poliestireno expandido) a una altura de $5 \mathrm{~cm}$ durante 6 min y luego fueron sumergidas en el nitrógeno líquido.

La descongelación se realizó sacando las pajillas del termo criogénico a las $3 \mathrm{~h}$ y se colocaron en baño María a $37^{\circ} \mathrm{C}$ por $30 \mathrm{~s}$, procediéndose a la evaluación de las motilidades individual y progresiva (Pérez et al., 2006).

\section{Análisis de Datos}

En el análisis de los parámetros espermáticos a la refrigeración y descongelación se utilizó un diseño completamente al azar con ocho repeticiones, donde el factor independiente fue el antioxidante Tempol en 4 concentraciones $(0,0.5,1.0$ y $1.5 \mathrm{mM})$. La diferencia entre medias se verificó con la prueba de Duncan. El análisis de datos se realizó con el software SAS v. 9.0.

\section{Resultados}

Las muestras colectadas de los conductos deferentes presentaron escasa viscosidad debido a la no presencia de secreciones de las glándulas bulbouretrales y de la próstata. En la evaluación inicial, la concentración espermática de la mezcla de muestras fue $19.6 \times 10^{6} / \mathrm{mm}^{3}$.

Se encontraron diferencias en la motilidad individual entre concentraciones de antioxidante Tempol en la etapa de refrigeración y en la etapa de congelación/descongelación ( $\mathrm{p}<0.05$ ). La motilidad aumentó en el grupo $0.5 \mathrm{mM}$ en ambos casos con respecto al control, pero disminuyó cuando se emplearon mayores cantidades del antioxidante (Cuadro 1).

En forma similar, el antioxidante afectó a la motilidad progresiva en ambas etapas de la criopreservación (Cuadro 2). Esto estaría indicando que los mayores efectos del antioxidante Tempol se darían en la etapa de enfriamiento de 10 a $5{ }^{\circ} \mathrm{C}$ y en el periodo de los 30 minutos de estabilización que en la etapa del congelamiento/descongelamiento. 
Cuadro 1. Motilidad individual (porcentaje \pm desviación estándar) de espermatozoides de alpaca colectados del conducto deferente, según etapa de criopreservación y niveles de antioxidante Tempol

\begin{tabular}{|c|c|c|c|c|c|}
\hline Etapa de criopreservación & & $\begin{array}{c}\text { Control } \\
(0.0 \mathrm{mM})\end{array}$ & $0.5 \mathrm{mM}$ & $1.0 \mathrm{mM}$ & $1.5 \mathrm{mM}$ \\
\hline Inicial & $80.6 \pm 4.4$ & & & & \\
\hline Refrigerado $^{1}$ & & $58.1^{\mathrm{b}} \pm 5.1$ & $63.7^{\mathrm{a}} \pm 3.5$ & $52.8^{\mathrm{b}} \pm 5.2$ & $45.0^{\mathrm{c}} \pm 4.6$ \\
\hline Congelado/descongelado & & $35.7^{\mathrm{b}} \pm 3.0$ & $42.3^{\mathrm{a}} \pm 3.8$ & $29.0^{c} \pm 6.5$ & $18.1^{\mathrm{d}} \pm 5.8$ \\
\hline
\end{tabular}

$a, b, c$ Diferentes superíndices dentro de filas denotan diferencia estadística $(p \varangle 0.05)$

${ }^{1}$ Corresponde a muestras con el antioxidante Tempol $\left(10^{\circ} \mathrm{C}\right)$

Cuadro 2. Motilidad progresiva (porcentaje \pm desviación estándar) de espermatozoides de alpaca colectados del conducto deferente, según etapa de criopreservación y niveles de antioxidante Tempol

\begin{tabular}{|c|c|c|c|c|c|}
\hline Etapa de criopreservación & & $\begin{array}{l}\text { Control } \\
(0.0 \mathrm{mM})\end{array}$ & $0.5 \mathrm{mM}$ & $1.0 \mathrm{mM}$ & $1.5 \mathrm{mM}$ \\
\hline Inicial & $63.5 \pm 5.2$ & & & & \\
\hline Refrigerado $^{1}$ & & $36.0^{\mathrm{b}} \pm 3.6$ & $42.4^{\mathrm{a}} \pm 3.5$ & $28.4^{\mathrm{b}} \pm 5.4$ & $21.7^{\mathrm{c}} \pm 5.9$ \\
\hline Congelado/descongelado & & $24.4^{\mathrm{b}} \pm 3.1$ & $27.3^{\mathrm{a}} \pm 4.6$ & $21.1^{\mathrm{c}} \pm 3.8$ & $15.8^{\mathrm{d}} \pm 4.2$ \\
\hline
\end{tabular}

a,b,c Diferentes superíndices dentro de filas denotan diferencia estadística $(p \varangle 0.05)$

${ }^{1}$ Corresponde a muestras con el antioxidante Tempol $\left(10^{\circ} \mathrm{C}\right)$

\section{Discusión}

\section{Motilidad Individual}

Entre las pocas referencias sobre procesamiento de semen proveniente del conducto deferente de la alpaca se encuentra el trabajo de Deza (2004), quien obtuvo altas motilidades individuales en la etapa de refrigeración (73\%) y valores inferiores en el caso de llama. Utilizando la misma metodología, Pérez et al. (2008) reportaron motilidades cercanas a 56.5 en dos alpacas, resultado similar al grupo control del presente estudio (58.1\%). Estas diferencias podrían deberse a la motilidad inicial, así como por las varia- ciones intra- e inter-especie de la calidad espermática (Nasrin, 2011). En este contexto, los hallazgos del estudio serían inferiores a los reportados por Deza (2004) y similares a los obtenidos por Pérez et al. (2008).

Se conoce poco acerca de la fisiología de los espermatozoides de la alpaca en condiciones de refrigeración. Es posible que los espermatozoides de alpaca respecto a la llama, tengan mejores condiciones para resistir los cambios de temperatura (Deza, 2004). En el presente estudio, la adición del antioxidante Tempol tendría efectos favorables cuando es aplicado en bajas dosis. Según Santiani et al. (2013), la adición del antioxidante Tempol al 
final de la curva de enfriamiento evitaría el daño celular por estrés oxidativo debido a la presencia de las especies reactivas de oxígeno en el semen.

Respecto a la motilidad individual de congelación/descongelación, Deza (2004) reportó $41 \%$ en alpacas y $35.8 \%$ en llamas, en ambos casos sin el uso de antioxidantes. El resultado fue muy similar al presente estudio con el uso de $0.5 \mathrm{mM}$ de antioxidante. Se desconocen los factores involucrados en la menor sensibilidad a la criopreservación de los espermatozoides de la llama respecto a la alpaca, pero es posible que estas diferencias se deban a las variaciones de la composición bioquímica y la función del plasma seminal que varía con la dieta y con el eyaculado dentro de machos, entre machos e interespecies (Nasrin, 2011). La ausencia del plasma seminal podría afectar el proceso de congelación/descongelación de espermatozoides, puesto que este componente del semen tendría factores que participan en la congelabilidad de los espermatozoides (Zahn et al., 2006). Al respecto, Kershaw-Young y Maxwell (2011) indican que altos niveles de plasma seminal en la incubación de espermas provocaría daño celular en los espermatozoides, y que solo la adición del $10 \%$ de plasma seminal facilitaría mejores resultados en la motilidad espermática de alpacas.

Según Mara et al. (2002), el antioxidante Tempol, un nitróxido estable a actividad SOD-simil (superoxidodismutasa), es permeable a las membranas celulares por su bajo peso molecular. Esta característica del antioxidante impediría la reducción de la toxicidad debida al oxígeno, y de los fenómenos de peroxidación de los lípidos que alteran las membranas celulares (Mitchell, 1990). De acuerdo con ello, Santiani et al. (2013) obtuvieron una mayor motilidad individual a la descongelación en el semen de alpaca colectado por vagina artificial cuando se adicionó Tempol $1.0 \mathrm{mM}$. Al parecer, la viscosidad del plasma seminal no permitiría una adecuada acción del antioxidante, por lo que sería necesario emplear mayores niveles.
Por otro lado, en estudios con espermatozoides epididimarios (desprovistos de plasma seminal) de testículos recuperados por castración (Banda et al., 2010) o en el camal (Santiani et al., 2010), utilizando dilutores en base a leche descremada, se encontró $17 \%$ de motilidad luego del proceso de descongelación, un valor superior al obtenido con dilutores en base a Tris. Sin embargo, en el presente estudio, con el dilutor en base a Tris se obtuvieron motilidades superiores, de allí que es posible que existan otros factores que explicarían estas variaciones.

En general, en trabajos previos, así como en el presente estudio, se han registrado una baja motilidad en los espermatozoides de la alpaca después del proceso de congelacióndescongelación, quedando por dilucidar los factores causales. Se conoce la existencia del estrés asociado a la congelación causado por los cambios de temperatura a que son sometidos los espermatozoides durante el proceso de enfriamiento, así como los efectos causados por el uso de crioprotectores y por la descongelación (Vishwanath y Shannon, 2000, Canorio y Paredes, 2012). El proceso de congelación del semen causa daños bioquímicos y funcionales a los espermatozoides, por lo que la fertilidad del semen congelado siempre es más baja comparada con el semen fresco (Leboeuf et al., 2000). El daño causado por las bajas temperaturas ocurre en la membrana plasmática, membrana acrosomal, mitocondrias y en la vaina del axonema (Salomón y Maxwell, 2000).

\section{Motilidad Progresiva}

En la refrigeración, la mayor motilidad progresiva de espermatozoides refrigerados (42.4\%) fue menor al $58.3 \%$ obtenido por Quintano (2002) en muestras refrigeradas hasta $6 \mathrm{~h}$; diferencias que podrían atribuirse al efecto individual (Nasrin, 2011). La disminución de la motilidad espermática se debería a la reducción de la temperatura, que permite reducir la actividad metabólica celular y aumentar el periodo de vida de las células (Medeiros et al., 2002). 
Los espermatozoides descongelados han tenido una motilidad progresiva de $27.3 \%$ con $0.5 \mathrm{mM}$ de Tempol, mayor que el control (24.4\%). Al respecto, Gonzáles (2008) encontró $20 \%$ de motilidad del tipo A o lineal en espermatozoides del epidídimo de la alpaca recuperados por lavado retrógrado. Es posible que la mayor motilidad encontrada se deba al método de colección, ya que podría tratarse de espermas maduros y con actividad fertilizante, donde el contacto directo con el medio ambiente ayudaría en la capacitación prematura de los espermatozoides desprovistos de plasma seminal (Katska et al., 1996; Vadnais et al., 2005). En la técnica de colección utilizada, los espermatozoides son expuestos brevemente al medio ambiente.

Se encontraron dos limitaciones en el trabajo. La primera fue que durante el tiempo de descanso poscolecta de muestras, la fistula del conducto deferente suele taponarse, y la segunda fue que se obtuvieron muestras de bajo volumen reduciendo el procesamiento a 3 o 4 pajillas por animal. Por otro lado, la ventaja observada fue que se puede disponer de muestras de espermatozoides en cualquier época del año y con buenos parámetros espermáticos.

\section{Conclusiones}

La adición del antioxidante Tempol a baja concentración $(0.5 \mathrm{mM})$ durante el proceso de enfriamiento de espermatozoides desprovistos de plasma seminal, mejora la motilidad individual y progresiva de refrigeración y congelación/descongelación. Mayores niveles del antioxidante afectan negativamente estos parámetros espermáticos.

\section{Agradecimientos}

Los autores agradecen a la Dirección de Investigación de la Universidad Nacional Micaela Bastidas de Apurímac por el financiamiento de este trabajo y a la Lic. Fanny Henao López por sus aportes en el logro del manuscrito.

\section{Literatura Citada}

1. Ball B, Medina V, Gravance CG, Baumbe J. 2001. Effect of antioxidants on preservation of motility, viability and acrosomal integrity of equine spermatozoa during storage at $5^{\circ} \mathrm{C}$. Theriogenology 56: 577-589. doi: 10.1016/S0093-691X(01)00590-8

2. Banda J, Evangelista S, Ruiz L, Sandoval R, Rodríguez C, Valdivia M, Santiani A. 2010. Efecto de dilutores en base a Tris, Tes y leche descremada en la criopreservación de espermatozoides obtenidos del epidídimo de alpaca. Rev Inv Vet Perú 21: 145-153. doi: 10.15381/rivep.v21i2.129

3. Bravo PW, Skidmore JA, Zhao XX. 2000. Reproductive aspects and storage of semen in camelidae. Anim Reprod Sci 62: 173-193. doi: 10.1016/S03784320(00)00158-5

4. Canorio N, Paredes F, Valdivia M. 2012. Cryocapacitation of alpaca (Vicugna pacos) sperm cell. Cryobiology 65: 364-366. doi:10.1016/ j.cryobiol.2012.07.077

5. Deza H. 2004. Conservación de espermatozoides obtenidos a través del conducto deferente en alpacas (Lama pacos) y llama (Lama glama) y su posterior viabilidad. Tesis de Médico Veterinario y Zootecnista. Puno: Univ Nacional del Altiplano. $71 \mathrm{p}$.

6. Foote R, Brockett C, Kaproth M. 2002. Motility and fertility of bull sperm in whole milk extender containing antioxidants. Anim Reprod Sci 71: 13-23. doi: 10.1016/S0378-4320(02)00018-0

7. Garnica J, Achata R, Bravo PW. 1993. Physical and biochemical characteristics of alpaca semen. Anim Reprod Sci 32: 85-90. doi: 10.1016/03784320(93)90059-Z

8. Gonzáles M. 2008. Obtención y criopreservación de espermatozoides de alpacas. Scientia 10: 223-234. 
9. Kershaw-Young CM, Maxwell WM. 2011. The effect of seminal plasma on alpaca sperm function. Theriogenology 76: 1197-1206. doi: 10.1016/j.theriogenology.2011.05.016

10. Katska L, Rynska B, Smor Z. 1996. Effect of seminal plasma on the in vitro fertilizability of bull spermatozoa. Anim Reprod Sci 44: 23-31. doi: 10.1016/03784320(96)01495-9

11. Leboeuf B, Restall B, Salamon S. 2000. Production and storage of goat semen for artificial insemination. Anim Reprod Sci 62: 113-141. doi: 10.1016/ S0378-4320(00)00156-1

12. Mara L, Accardo C, Dattena M, Branca A, Casu S, Cappai P. 2002. Efecto de un antioxidante en la conservación de semen ovino. Resultados preliminares. En: XXVII Jornadas Científicas y VI Jornadas Internacionales de la Sociedad Española de Ovinotecnia y Caprinotecnia. Valencia, España.

13. Medeiros CM, Forell F, Oliveira AT, Rodrigues JL. 2002. Current status of sperm cryopreservation: why isn't it better? Theriogenology 57: 327-344. doi: 10.1016/S0093-691X(01)00674-4

14. Mitchell J, Samuni A, Krishna M, Degraff W, Ahn M, Samuni U, Russo A. 1990. Biologically active metalindependent superoxide dismutase mimics. Biochemistry 29: 2802-2807. doi: $10.1021 / \mathrm{bi00463a024}$

15. Morton K, Bathgate R, Evans G, Maxwell WMC. 2007. Cryopreservation of epididymal alpaca (Vicugna pacos) sperm: a comparison of citrate, Tris- and lactose-based diluents and pellets and straws. Reprod Fertil Dev 19: 792-796. doi: 10.1071/RD07049

16. Morton KM, Evans G, Maxwell WMC. 2010. Effect of glycerol concentration, Equex STM ${ }^{\circledR}$ supplementation and liquid storage prior to freezing on the motility and acrosome integrity of frozen-thawed epididymal alpaca (Vicugna pacos) sperm. Theriogenology 74: 311-316. doi: 10.1016 / j.theriogenology.2010.02.015.
17. Nasrin S. 2011. Protein profiles and biochemical characteristics of semen: influence on frozen-thawed spermatozoal quality in rams (Ovis aries) and alpacas (Vicugna pacos). PhD Thesis. Italy: Universita Degli studi Di Padova. $208 \mathrm{p}$.

18. Niasari-Naslaji A, Mosaferi S, Bahmani N, Gerami A, Gharahdaghi AA, Abarghani A, Ghanbari A. 2007. Semen cryopreservation in bactrian camel (Camelus bactrianus) using SHOTOR diluent: effects of cooling rates and glycerol concentrations. Theriogenology 68: 618-625. doi: 10.1016/ j.theriogenology.2007.04.059

19. Parks JE, Graham JK. 1992. Effects of cryopreservation procedures on sperm membranes. Theriogenology 38: 209222. doi: 10.1530/REP-07-0118

20. Pérez G, Quispe T, Olivera L, Zevallos $J$, Pérez U. 2008. Producción espermática del conducto deferente en alpacas durante la época reproductiva. En: Resumen I Simposium Internacional en Camélidos Sudamericanos. CuscoPerú: Univ Nacional San Antonio Abad del Cusco.

21. Pérez MG, Apaza E, Deza H. 2006. Congelación de los espermatozoides procedentes de los conductos deferentes de camélidos. Allpaqa 11(1): 17-23.

22. Quintano J. 2002. Determinación de la sobrevivencia de los espermatozoides de alpaca (Lama pacos) colectados del conducto deferente con el uso de tres dilutores. Tesis de Médico Veterinario y Zootecnista. Puno: Univ Nacional del Altiplano. $58 \mathrm{p}$.

23. Rota A, Strom B, Fosberg CL, Rodríguez-Martínez H. 1997. Effects of Equex STM paste on viability of frozen-thawed dog spermatozoa during in vitro incubation at $38{ }^{\circ} \mathrm{C}$. Theriogenology 47: 1093-1101. doi: 10.1016/ S0093-691X(97)00066-6

24. Ruiz L, Santiani A, Sandoval R, Huanca W, Delgado A, Coronado L, Alzamora C. 2007. Efecto de dos antioxidantes (Tempo y Tempol) en la 
criopreservación de semen ovino empleando un dilutor en base a tris. Rev Inv Vet Perú 18: 99-106. doi: 10.15381/ rivep.v18i2.1284

25. Salamon S, Maxwell WM. 2000. Storage of ram semen. Anim Reprod Sci 62: 77-111. doi: 10.1016/S03784320(00)00155-X

26. Sandoval R, Santiani A, Ruiz L, Leyva V, Coronado L, Delgado A. 2007. Criopreservación de semen ovino empleando tres dilutores y cuatro combinaciones de agentes crioprotectores permeantes y no permeantes. Rev Inv Vet Perú 18: 107-114. doi: 10.15381/ rivep.v18i2.1285

27. Santiani A, Evangelista $S$, Cheuquemán $C$, von Baer $A$, Risopatrón J, Sánchez R. 2012. Evaluación de la integridad de ADN mediante citometría de flujo en espermatozoides de alpaca criopreservados con análogos de superóxido dismutasa. Rev Inv Vet Perú 23: 182-191. doi: 10.15381/ rivep.v23i2.898

28. Santiani A, Evangelista S, Sepúlveda $N$, Risopatrón J, Villegas, Sánchez $R$. 2014. Addition of superoxide dismutase mimics during cooling process prevents oxidative stress and improves semen quality parameters in frozen/thawed ram spermatozoa. Theriogenology 82: 884889. doi: $10.1016 / \mathrm{j}$.theriogenology.2014.07.002

29. Santiani A, Evangelista S, Valdivia M, Risopatrón J, Sánchez R. 2013. Effect of the addition of two superoxide dismutase analogues (Tempo and Tempol) to alpaca semen extender for cryopreservation. Theriogenology 79 : 842-846. doi: 10.1016/j.theriogenology.2012.12.012
30. Santiani A, Huanca W, Sapana R, Huanca T, Sepúlveda N, Sánchez R. 2005. Effects on the quality of frozenthawed alpaca (Lama pacos) semen using two different cryoprotectants and extenders._Asian J Androl 7: 303-309. doi: 10.1111 / j.1745-7262.2005.00021.x

31. Santiani A, Rodríguez, J, Evangelista S, Valdivia M. 2010. Cryopreservation of epididymal alpaca (Vicugna pacos) sperm. Reprod Biomed Online 20 (Suppl 2): S56-S57. doi: 10.1016/S14726483(10)62402-9

32. Sumar J. 1996. Reproduction in llamas and alpacas. Anim Reprod Sci 42: 405415. doi: 10.1016/0378-4320(96)01538-2

33. Tortolero I, Arata-Bellabarba G, Osuna JA, Gómez R, Regadera J. 2005. Estrés oxidativo y función espermática. Revisión. Rev Venez Endocrinol Metab 3: 12-19.

34. Vadnais ML, Kirkwood RN, Tempelman RJ, Sprecher DJ, Chou $K$. 2005. Effect of cooling and seminal plasma on the capacitation status of fresh boar sperm as determined using chlortetracycline assay. Anim Reprod Sci 87: 121-132. doi: 10.1016/j.anireprosci.2004.10.004

35. Vishwanath R, Shannon P. 2000. Storage of bovine semen in liquid and frozen state. Anim Reprod Sci 62: 23-53. doi: 10.1016/S0378-4320(00)00153-6

36. von Baer A, Hellemann C. 1998. Semen characteristics in the llama (Lama glama). Arch Med Vet 30: 171-176.

37. Zahn FS, Papa FO, Melo CM. 2006. Blood serum, seminal plasma and sperm membrane protein profiles in stallions: are they correlated to semen freezability? Anim Reprod Sci 94: 64-66. doi: 10.1016/ j.anireprosci.2006.03.088 